\title{
An Efficient Brain Tumor Detection Algorithm Using Watershed \& Thresholding Based Segmentation
}

\author{
Anam Mustaqeem \\ Department of Software Engineering, UET Taxila \\ E-mail: anam846@yahoo.com \\ Ali Javed \\ Department of Software Engineering, UET Taxila \\ E-mail: ali.javed@uettaxila.edu.pk \\ Tehseen Fatima \\ Department of Computer Engineering, UET Taxila \\ E-mail: tehseen_2k6@yahoo.com
}

\begin{abstract}
During past few years, brain tumor segmentation in magnetic resonance imaging (MRI) has become an emergent research area in the field of medical imaging system. Brain tumor detection helps in finding the exact size and location of tumor. An efficient algorithm is proposed in this paper for tumor detection based on segmentation and morphological operators. Firstly quality of scanned image is enhanced and then morphological operators are applied to detect the tumor in the scanned image.
\end{abstract}

Index Terms - Brain Tumor, MRI, Morphological Operators, Segmentation

\section{INTRODUCTION}

In medical imaging, 3D segmentation of images plays a vital role in stages which occur before implementing object recognition. 3D image segmentation helps in automated diagnosis of brain diseases and helps in qualitative and quantitative analysis of images such as measuring accurate size and volume of detected portion.

Accurate measurements in brain diagnosis are quite difficult because of diverse shapes, sizes and appearances of tumors. Tumors can grow abruptly causing defects in neighboring tissues also, which gives an overall abnormal structure for healthy tissues as well. In this paper, we will develop a technique of 3D segmentation of a brain tumor by using segmentation in conjunction with morphological operations.

\section{A. Tumor:}

The word tumor is a synonym for a word neoplasm which is formed by an abnormal growth of cells Tumor is something totally different from cancer.

\section{1) Types of Tumor:}

There are three common types of tumor: 1)Benign; 2)
Pre-Malignant;3)Malignant(cancer can only be malignant).[1]

a) Benign Tumor: A benign tumor is a tumor is the one that does not expand in an abrupt way; it doesn't affect its neighboring healthy tissues and also does not expand to non-adjacent tissues. Moles are the common example of benign tumors.

b) Pre-Malignant Tumor: Premalignant Tumor is a precancerous stage, considered as a disease, if not properly treated it may lead to cancer.

c) Malignant Tumor: Malignancy (mal- = "bad" and ignis = "fire") is the type of tumor, that grows worse with the passage of time and ultimately results in the death of a person. Malignant is basically a medical term that describes a severe progressing disease. Malignant tumor is a term which is typically used for the description of cancer.

\section{B. Magnetic Resonance Imaging (MRI):}

MRI is basically used in the biomedical to detect and visualize finer details in the internal structure of the body. This technique is basically used to detect the differences in the tissues which have a far better technique as compared to computed tomography. So this makes this technique a very special one for the brain tumor detection and cancer imaging. [2]

CT uses ionizing radiation but MRI uses strong magnetic field to align the nuclear magnetization then radio frequencies changes the alignment of the magnetization which can be detected by the scanner. That signal can be further processed to create the extra information of the body.

This research paper is divided in six parts, in the second part analysis and findings are discussed. In the third portion related work is explained, many 
researchers are currently working in this field. Many related research papers are properly explained in this region. Forth portion is proposed methodology, all the phases of our proposed system is explained in details. All the stages including preprocessing, processing and post processing are discussed there. In the next section all experimental results are shown with proper diagrams and figures. Then in the last section conclusion and future work is discussed.

\section{ANALYSIS AND FINDINGS}

Now days, one of the main cause for increasing mortality among children and adults is brain tumor. It has been concluded from the research of most of the developed countries that number of people suffering and dying from brain tumors has been increased to 300 per year during past few decades.

Bar Graph is given below, showing number of persons who have diagnosed from tumor and number of persons who died of tumor.

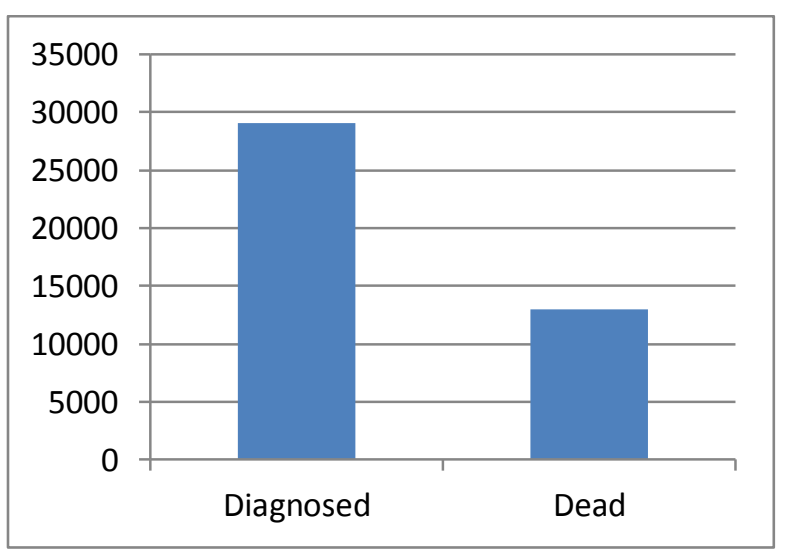

Figure 1: Analysis of Tumor Diagnosis [9]

Pie chart is given below, which shows rate of tumor diagnosis in some countries.

\section{Brain Tumors Diagnosed}

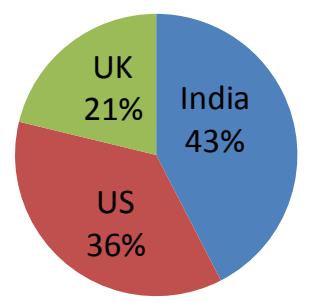

Figure 2: Rate of Tumor Diagnosis Per Year[9]

\section{RELATED WORK}

Segmentation is the process where an image is divided into the different regions on some similarity bases. Basic function of the segmentation is that we can easily extract information and different features from the images. As brain tumor detection is a very time consuming process which is done by medical experts. So to tackle this issue many segmentation techniques are developed by the image processing experts. [3] Many of these techniques are not properly defined rather they are ad hoc techniques.

Some of the most common methods are: 1) Amplitude thresholding; 2) Texture segmentation; 3) Template matching; 4) Region-growing segmentation

When experts work on tumor images then they use three different types of algorithms. Some of the techniques based on pixel based, some based on texture of images and some of them based on structure of images. Gopal,N.N. Karnan, M. [4] suggested an algorithm which used multi-scale image segmentation, this algorithm was based on fuzzy c-mean algorithm for the detection of brain tumor. Joshi, D.M.; Rana, N.K.; Misra, V.M. [5] suggested an improved technique for tumor detection, this algorithm used neuro fuzzy technique for the segmentation for the tumor detection.

Ming niwu,chia-chen Lin and chin-chenchang[6], proposed an algorithm which uses a clustering technique(k-means) to detect the brain tumor in MR images. First of all they convert the gray scale images into color images and then by the help $\mathrm{k}$ means clustering.

Hossam M. Moftah, Aboul Ella Hassanien, and MohamoudShoman [7], they used k mean algorithm with connected component labeling. Clustering can be done with the help of object rendering process in $2 \mathrm{D}$ slices and then $3 \mathrm{D}$ patch is obtained.

P.Vasuda, S.Satheesh [8], proposed a technique to detect tumors from MR images suing fuzzy clustering technique. This algorithm uses fuzzy $\mathrm{C}$-means but the major drawback of this algorithm is the computational time required. This paper also compares the FCM and improved version of FCM.

\section{Proposed Methodology}

\section{A. Image Acquisition:}

Images are obtained using MRI scan and these scanned images are displayed in a two dimensional matrices having pixels as its elements. These matrices are dependent on matrix size and its field of view. Images are stored in MATLAB and displayed as a gray scale image of size $256 * 256$. The entries of a gray scale image are ranging from 0 to 255 , where 0 shows total black color and 255 shows pure white color. Entries between this range vary in intensity from black to white.

For experimental purpose 30 female and 30 male patients were examined, all patients have ages ranging from 20 to 60 years. Their MRI scans were stored in database of images in JPEG image formats. 


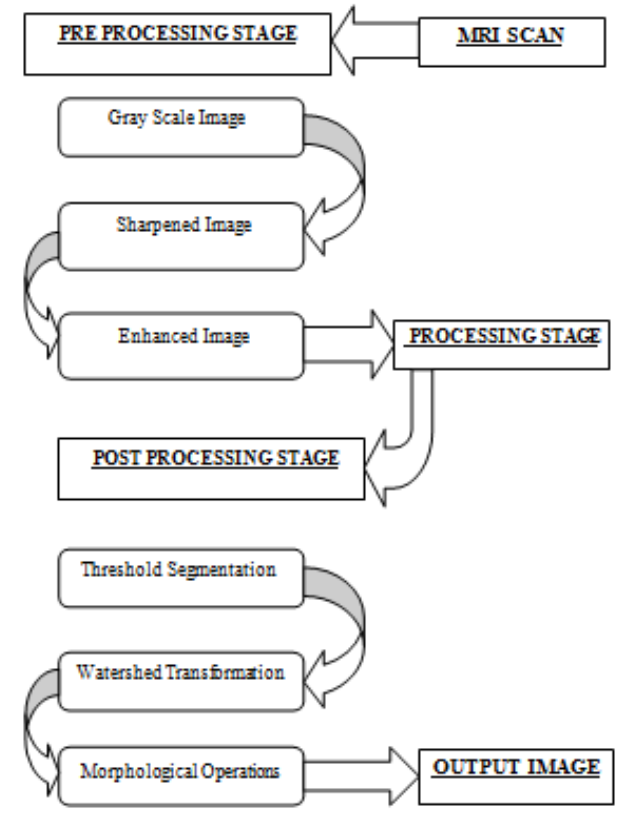

Figure3: Stages of Tumor Detection

\section{B. Preprocessing:}

In this phase image is enhanced in the way that finer details are improved and noise is removed from the image. Most commonly used enhancement and noise reduction techniques are implemented that can give best possible results. Enhancement will result in more prominent edges and a sharpened image is obtained, noise will be reduced thus reducing the blurring effect from the image. In addition to enhancement, image segmentation will also be applied. This improved and enhanced image will help in detecting edges and improving the quality of the overall image. Edge detection will lead to finding the exact location of tumor. Following steps will be followed in the preprocessing stage:

The acquired MRI scanned image, stored in database is converted to gray scale image of size $255 * 255 ; 2$ ) image is processed to remove any noise represent. Visual quality of noisy image will not be satisfactory; 3) the noiseless, high quality image is then operated by a high pass filter for sharpening and edge detection. ; 4) The obtained sharpened image is then added to original image for further enhancement.

a) Noise Removal: Many filters are used to remove the noise from the images. Linear filters can also serve the purpose like Gaussian, averaging filters. For example average filters are used to remove salt and pepper noise from the image. Because in this filter pixel's value is replaced with its neighborhood values.

Median filter is also used to remove the noise like salt and pepper and weighted average filter is the variation of this filter and can be implemented easily and give good results. In the median filter value of pixel is determined by the median of the neighboring pixels. This filter is less sensitive than the outliers.

b) Image Sharpening: Sharpening of the image can be achieved by using different high pass filters. As now noise is been removed by using different low pass filters, we need to sharpens the image as we need the sharp edges because this will help us to detect the boundary of the tumor.

Gaussian high pass filter is used to enhance the boundaries of the objects in the image. Gaussian filter gives very high rated results and used very widely to enhance the finer details of the objects.

\section{Processing:}

1) Segmentation: Image segmentation is based on the division of the image into regions. Division is done on the basis of similar attributes. Similarities are separated out into groups. Basic purpose of segmentation is the extraction of important features from the image, from which information can easily be perceived. Brain tumor segmentation from MRI images is an interesting but challenging task in the field of medical imaging.

\section{Post-Processing:}

In processing segmentation is done using following methods.

1) Threshold Segmentation: Threshold segmentation is one of the simplest segmentation methods. The input gray scale image is converted into a binary format. The method is based on a threshold value which will convert gray scale image into a binary image format. The main logic is the selection of a threshold value. Some common methods used under this segmentation include maximum entropy method and k- means clustering method for segmentation.[10]

2) Watershed Segmentation: It is one of the best methods to group pixels of an image on the basis of their intensities. Pixels falling under similar intensities are grouped together. It is a good segmentation technique for dividing an image to separate a tumor from the image Watershed is a mathematical morphological operating tool. Watershed is normally used for checking output rather than using as an input segmentation technique because it usually suffers from over segmentation and under segmentation.[11]

For using watershed segmentation different methods are used. Two basic principle methods are given below: 1) the computed local minima of the image gradient are chosen as a marker. In this method an over segmentation occurs. After choosing marker region merging is done as a second step; 2) Watershed transformation using markers utilizes the specifically defined marker positions. These positions are either defined 
explicitly by a user or they can be determined automatically by using morphological tools.

3) Morphological Operators: After converting the image in the binary format, some morphological operations are applied on the converted binary image. The purpose of the morphological operators is to separate the tumor part of the image. Now only the tumor portion of the image is visible, shown as white color. This portion has the highest intensity than other regions of the image.

Some of the commands used in morphing are given below: 1) Strel: Used for creating morphological structuring element; 2) Imerode():Used to erode (Shrink) an image.[12] ;3)Imdilate():Used for dilating (filling, expanding) an image.[12]

\section{EXPERIMENTAL OUTCOMES}

First of all input image is shown here, figure 4 shows input images which has brain tumor. Threshold segmentation is applied on these images which contains brain tumor. The results are shown in the figure 5 .
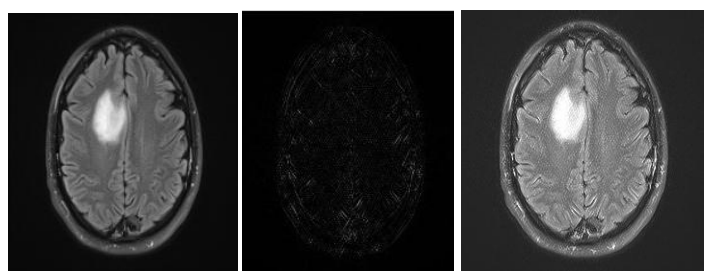

Figure 4: Input Brain Image

In the following figure white spot is shown, which is the result of threshold segmentation applied on the images. This is basically the area with the intensity values higher than the defined threshold. High intensity areas mostly comprises of tumors. So through threshold segmentation we can specify the location of tumor.

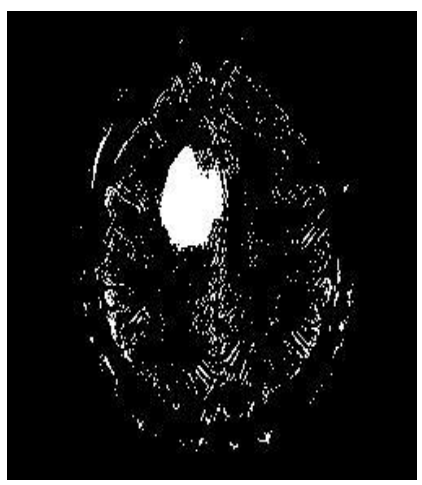

Figure 5: Threshold Segmentation

Then there is a technique called watershed segmentation which is applied on the resulted image obtained after threshold segmentation. Only the portion which contains tumor is highlighted here. The portions with the high intensity values are detected through threshold segmentation. These portions are marked through watershed segmentation methods.

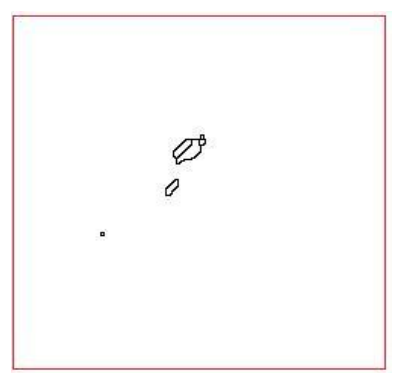

Figure 6: Watershed Segmentation

Morphological operators are applied after the watershed segmentation. The results are quite prominent and this technique is very efficient.
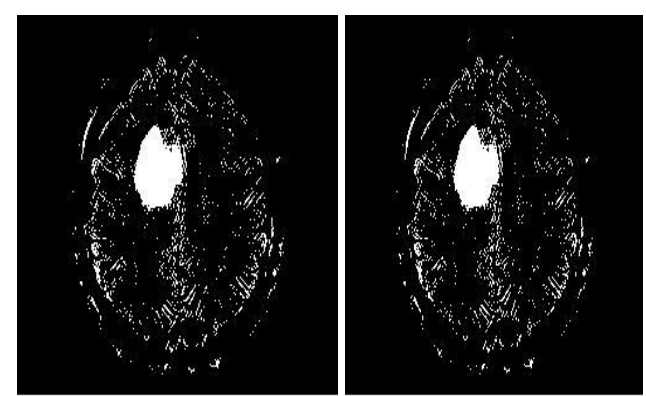

Figure 7: Morphological Operators

Figure 8 contains the resulted image obtained after applying functions like erosion.

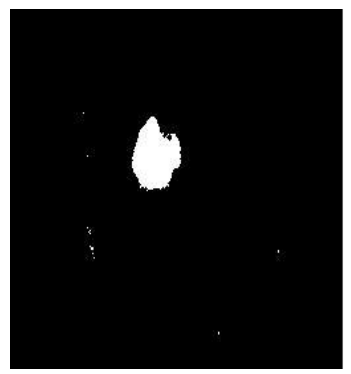

Figure 8: Imerode With strel('disk',1)

Figure 9 contains the resulted image of dilation function which is applied after the erosion.

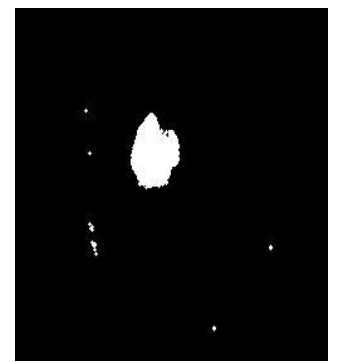

Figure 9: Imdilate With strel('disk',1)

\section{A. Output Image:}

Tumor is displayed as white portion in the image. 

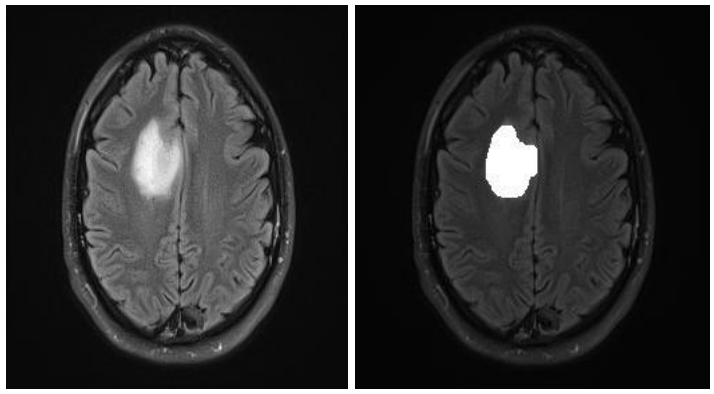

Figure 10: Tumor Detected As White Portion

\section{CONCLUSION AND FUTURE WORK}

This research was conducted to detect brain tumor using medical imaging techniques. The main technique used was segmentation, which is done using a method based on threshold segmentation, watershed segmentation and morphological operators. The proposed segmentation method was experimented with MRI scanned images of human brains: thus locating tumor in the images. Samples of human brains were taken, scanned using MRI process and then were processed through segmentation methods thus giving efficient end results.

This technique gives efficient results as compared to previous researches. Experiments are applied on various images and results were extraordinary. Our proposed research is easy to execute and thus can be managed easily.

Our future work is to extend our proposed method for color based segmentation of 3D images. For this purpose we need a classification method to organize three dimensional objects into separate feature classes, whose characteristics can help in diagnosis of brain diseases.

\section{ACKNOWLEDGMENT}

We owe deep appreciation and gratitude to our supervisor, as it was a great privilege for us to carry out the research under the supervision of Engr. Ali Javed. We are very thankful to all those who helped us in collecting data for brain tumors.

\section{REFERENCES}

[1] Oelze, M.L,Zachary, J.F. , O'Brien, W.D., Jr., "Differentiation of tumor types in vivo by scatterer property estimates and parametric images using ultrasound backscatter " , on page(s) :1014 - 1017 Vol.1, 5-8 Oct. 2003.

[2] Devos, A, Lukas, L., "Does the combination of magnetic resonance imaging and spectroscopic imaging improve the classification of brain tumours?", On Page(s): 407 - 410, Engineering in Medicine and Biology Society, 2004. IEMBS '04. 26th Annual International Conference of the IEEE, 1-5 Sept. 2004.
[3] Farmer, M.E, Jain, A.K. , "A wrapper-based approach to image segmentation and classification", Page(s): 2060 - 2072 , Image Processing, IEEE Transactions on journals and magazines, Dec. 2005.

[4] Gopal,N.N. Karnan, M. , "Diagnose brain tumor through MRI using image processing clustering algorithms such as Fuzzy C Means along with intelligent optimization techniques “, Page(s): 1 4, Computational Intelligence and Computing Research (ICCIC), 2010 IEEE International Conference, 28-29 Dec. 2010.

[5] Joshi, D.M.; Rana, N.K.; Misra, V.M. i ," Classification of Brain Cancer using Artificial Neural Network “, Page(s): 112 - 116, Electronic Computer Technology (ICECT), 2010 International Conference, 7-10 May 2010

[6] Ming niwu,chia-chen Lin and chin-chenchang, "Brain Tumor Detection Using Color-Based KMeans Clustering Segmentation", Page(s): 245 250 , Intelligent Information Hiding and Multimedia Signal Processing, 2007. IIHMSP 2007. Third International Conference,26-28 Nov. 2007

[7] Hossam M. Moftah, Aboul Ella Hassanien, Mohamoud Shoman, "3D Brain Tumor Segmentation Scheme using K-mean Clustering and Connected Component Labeling Algorithms", Page(s): 320 - 324 , Intelligent Systems Design and Applications (ISDA), 2010 10th International Conference, Nov. 29 2010-Dec. 12010

[8] P.Vasuda, S.Satheesh, "Improved Fuzzy C-Means Algorithm for MR Brain Image Segmentation", Page(s): 1713-1715, (IJCSE) International Journal on Computer Science and Engineering, Vol. 02, 05, 2010.

[9] T. Logeswari, M. Karnan, "An improved implementation of brain tumor detection using segmentation based on soft computing", Page(s): 006-014, Journal of Cancer Research and Experimental Oncology Vol. 2(1), March 2010.

[10] Jichuan Shi, "Adaptive local threshold with shape information and its application to object segmentation", Page(s)1123 - 1128, Robotics and Biomimetics (ROBIO), 2009 IEEE International Conference, 19-23 Dec. 2009.

[11] Gang Li , "Improved watershed segmentation with optimal scale based on ordered dither halftone and mutual information", Page(s) 296 - 300, Computer Science and Information Technology (ICCSIT), 2010 3rd IEEE International Conference ,9-11 July 2010.

[12] Hedberg,H., Kristensen, F. ,Nilsson, P. ,Owall, V., "A low complexity architecture for binary image erosion and dilation using structuring element decomposition", Page(s): 3431 - 3434 Vol. 4 , 
Circuits and Systems, 2005. ISCAS 2005. IEEE International Symposium ,23-26 May 2005.

Engr. Anam Mustaqeem, is MSc Scholar in Department of Software Engineering at University of Engineering and Technology Taxila. She completed her Bachelor's degree in Software Engineering from University of Engineering and Technology Taxila in 2010 .

She has done extraordinary work in her four years degree of engineering. She is currently working on multimedia transmission over vehicular adhoc networks (VANETs). Her areas of interest are digital image processing, Medical Imaging, Software Quality Assurance, Wireless Networks and Adhoc Networks. She is trying hard to bring innovation in above mentioned fields.

Engr. Ali Javed, is a $\mathrm{PhD}$ Scholar in Department of Computer Engineering at University of Engineering \& Technology Taxila. He is serving as an Assistant Professor in the Department of Software Engineering, University of Engineering and Technology Taxila, Pakistan. He accomplished his M.Sc in Computer Engineering from University of Engineering and Technology Taxila, Pakistan in February, 2010. His areas of interest are Video Summarization, Digital Image Processing, Computer vision, Software Quality Assurance, Software testing and Software Requirements Analysis.

Engr. Tehseen Fatima, is MSc student in Department of Computer Engineering at University of Engineering and Technology Taxila. She completed her Bachelor's degree in Computer Engineering from University of Engineering and Technology Taxila in 2010. She is currently working as research associate at Mirpur University of Sciences and Technology. Her areas of interest are Digital Image Processing, Operating Systems and Programming Fundamentals. 